\title{
Helical Electronic Transitions of Spiroconjugated Molecules
}

\author{
Marc H. Garner a and Clemence Corminboeuf*a
}

Received 00th January 20xx, Accepted 00th January 20xx

DOI: $10.1039 / x 0 x \times 00000 x$

The two $\pi$-systems of allene can mix into helical molecular orbitals (MOs), yet the helicity is lost in the $\pi-\pi^{*}$ transitions. In spiroconjugated molecules the relative orientation of the two $\pi$ systems is different as only half the $\pi$-MOs become helical. Consequently, the helicity of the electronic transitions is symmetry protected and thus helical $\pi$-conjugation can manifest in observable electronic and chiroptical properties.

Allenes, cumulenes, and polyynes have two perpendicular $\pi$ systems, which lie in each their plane. ${ }^{1,2}$ One may intuitively expect the $\pi$-electrons to be separately delocalized in the molecular planes. However, the two $\pi$-systems can mix, and consequently the electrons may not be restricted to the planes. In allene (Scheme 1), the orbital-mixing is mediated by the $p$ orbitals on its central $s p$-hybridized carbon as shown in Figure 1. Whenever we consider these two p-orbitals, an initial choice is made for their orientation. ${ }^{3-5}$ By convention, we orient allene relative to its two mirror-planes. Shown in Figure $1 a$, the $p_{x}$ and $p_{y}$ basis functions will each have optimal overlap to one end of the molecule. However, the $p_{x}$ and $p_{y}$ basis functions can be rotated $45^{\circ}$ around the allenic axis by making linear combinations of the two as shown in Figure 1b. This forms a socalled coarctate orbital system, which means there is continuous $\pi$-overlap between the carbon termini despite their $90^{\circ}$ relative orientation. ${ }^{3,} 6$ The choice between these two orientations is arbitrary. ${ }^{3,4}$

Spiro-
Scheme 1. Overview of molecules

\footnotetext{
a. Laboratory for Computational Molecular Design, Institute of Chemical Sciences and Engineering, Ecole Polytechnique Federale de Lausanne (EPFL), 1015

Lausanne, Switzerland.E-mail: clemence.corminboeuf@epfl.ch.

+ Electronic Supplementary Information (ESI) available: Computational Details, Change of density schematics, Silicon and Germanium analogues, Cyclically-linked molecules, Spirononatetraene, Tricyclic spiro-analogues.
}

More recently, Honda et al., synthesized a radical cation of spiropentasiladiene (Scheme 1) and found that spin and charge can delocalize between its two perpendicular $\pi$-systems. ${ }^{7}$ Let us consider the carbon-analogue spiropentadiene (Scheme 1). Its two $\pi$-systems are connected through a formally saturated spiro-carbon. ${ }^{8}, 9$ Shown in Figure $1 \mathrm{~b}$, we can make the same orbital rotation in spiropentadiene as we did for allene. The $p$ orbitals on the formally saturated spiro-carbon will participate in the $\sigma$-bonds of the molecule and are normally considered in the form of $s p$-hybrid orbitals. However, these $p$-orbitals are mathematically part of the carbon basis set and are available as relay orbitals for the through-bond coupling between the two $\pi$-systems. In similar fashion, $\sigma$-orbitals mediate through-bond coupling between the perpendicular $\pi$-systems in larger tricyclic analogues of spiroconjugated molecules (Scheme 1) as described by Gleiter and co-workers. ${ }^{10,11}$

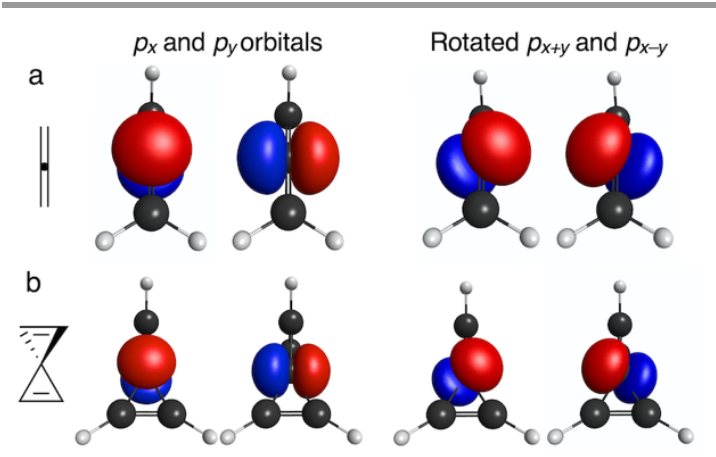

Figure 1. $p$-orbitals of the central carbon atom represented in two different rotations of allene (a) and spiropentadiene (b).

In chiral allenes, cumulenes, and polyynes the electron delocalization between the two $\pi$-systems is expressed in the form of helical MOs. ${ }^{3}, 12,13$ This helical $\pi$-conjugation is also called the electrohelicity effect. While the molecules where this effect appears are single-handed chiral, both helicities are present in the electronic structure. The chirality associated with the electronic structure is thus more complex than that of the molecular structure. ${ }^{14}$ The optical activity of odd-carbon 
cumulenes is limited by the near-degeneracy of their helical MOs. ${ }^{15}$ Although the helical HOMO and HOMO-1 can be split energetically by substituents, ${ }^{15-17}$ it seems that the dual-helicity limits the possibility of experimentally observable effects arising from the MO helicity. Still, relations to molecular properties have been proposed.12, ${ }^{18-23}$ Given that the through-bond electron delocalization in spiroconjugated molecules is closely related to that in allene (Figure 1), an interesting question is whether different types of $\pi$-systems are also limited by the dual-helicity of the MOs?
Here we describe the helical $\pi$-conjugation in allene and spiroconjugated molecules. We demonstrate that in the latter both the electronic structure and $\pi-\pi^{*}$ transitions have distinct helicity because it is symmetry protected. We revisit the $\pi-\pi *$ transitions of allene derivates, which are the simplest species with helical MOs. Due to the symmetry of allene its electronic transitions are limited by the dual-helicity of the MOs, and we examine why spiroconjugated molecules do not suffer from this limitation. Finally, we discuss the experimental implications of optical transitions with helical change of electron density and high rotatory strengths that correlate with the helicity.
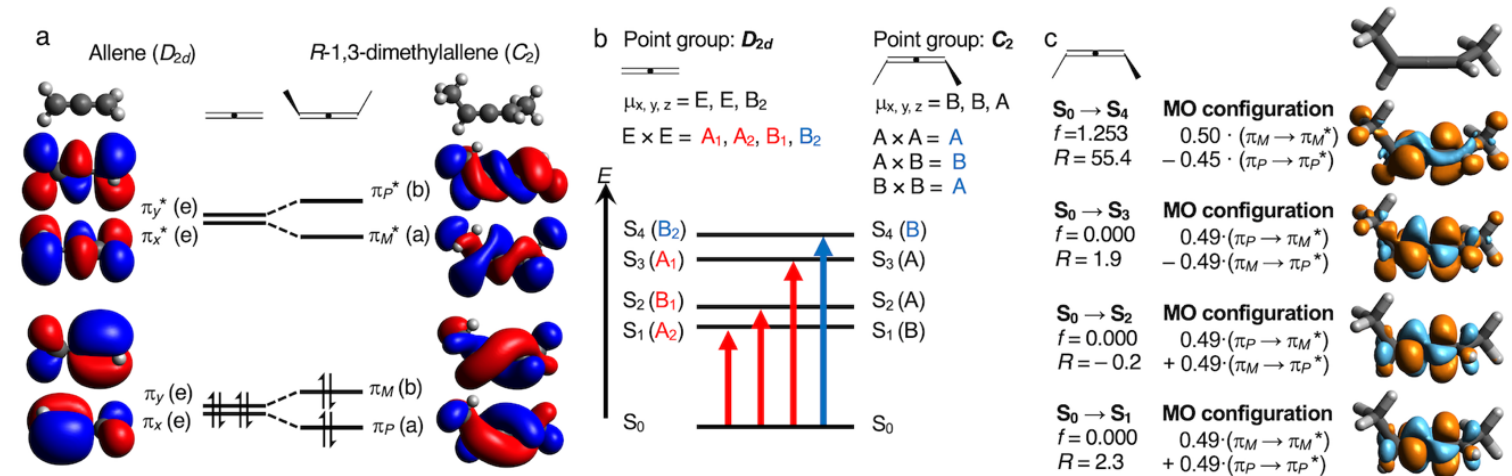

Figure 2. a) HOMO-1 to LUMO+1 of allene and $R$-1,3-dimethylallene. b) Overview of their first four electronic transitions, and their symmetry designations. Electric-dipole $\left(\mu_{x, y, z}\right)$ allowed transitions are in blue. c) Change of electron density for the $\mathrm{S}_{0} \rightarrow \mathrm{S}_{1}, \mathrm{~S}_{0} \rightarrow \mathrm{S}_{2}, \mathrm{~S}_{0} \rightarrow \mathrm{S}_{3}$ and $\mathrm{S}_{0} \rightarrow \mathrm{S}_{4}$ transitions of $R$-1,3-dimethylallene. Oscillator strengths, $f$, are dimensionless. Rotatory strengths, $R$, are in $10^{40} \mathrm{erg} \cdot \mathrm{esu} \cdot \mathrm{cm} / \mathrm{Gauss}$. Depletion of density is in orange. Computed at the $\omega \mathrm{B} 97 \mathrm{X}-\mathrm{D} / \mathrm{Def2}-\mathrm{TZVP}$ level of theory

Allene is routinely described as having two orthogonal $\pi$ systems lying in each their plane. ${ }^{1,2}$ The highest occupied MO (HOMO) and HOMO-1 are degenerate $\pi$-orbitals as shown in Figure 2a. However, when the symmetry of the molecule is reduced from $D_{2 d}$ to $C_{2}$ by substituting two of the hydrogens, the $\pi$-systems mix and helical $\pi$-MOs are formed. ${ }^{3,12}$ In the case of $R$-1,3-dimethyallene the HOMO is an M-helix and the HOMO1 is a $P$-helix. In $D_{2 d}$-allene, the resulting $\pi \rightarrow \pi *$ transitions between the two degenerate occupied and two degenerate unoccupied $\pi$-MOs consist of the four possible linear combinations of excitations between the two sets of MOs. The four transitions are non-degenerate and belong to $A_{2}, B_{1}, A_{1}$, and $B_{2}$ irreducible representations as outlined in Figure $2 b .{ }^{24}$ Only the $S_{0} \rightarrow S_{4}\left(B_{2}\right)$ transition is allowed (same irreducible representation as $\left.\mu_{x, y, z}\right)$. The substituents change little, and the first three transitions remain quasi-forbidden in dimethylallene. The effect of substituents and solvents on the optical properties of allene has been studied in great detail, and we refer to the extensive literature for analysis of their spectra. ${ }^{15,}$ 25-27 The transitions of dimethylallene are almost equally-weighted combinations of excitations from occupied $\pi_{P / M}$ to unoccupied $\pi_{P / M^{*}}$. Oscillator strengths and the specific $M O$ weights will be method dependent, see computational details in ESI part A.

Figure $2 c$ shows the change of electron density during the $\pi \rightarrow \pi^{*}$ transitions of $R-1,3$-dimethylallene. The change of density appears primarily in the two planes of the molecule and there is this little indication of mixing between the two $\pi$ systems. We attribute this lack of helicity to the dual-helicity of the HOMO and HOMO- 1 . The four $\pi \rightarrow \pi^{*}$ transitions of dimethyl allene are superpositions of helical MO excitations (see schematic overview in Figure S4 and S5). Any individual excitation involving helical MOs yields a helical pattern in the change of electron density; however, transitions between their linear combinations show a linear pattern.

In spiroconjugated molecules, two $\pi$-systems are separated by an $s p^{3}$-hybridized carbon atom. ${ }^{8}, 9$ The two $\pi$-systems are oriented $90^{\circ}$ relative to each other and interact by a welldescribed through-space interaction. ${ }^{28}$ Still, the $\pi$-electrons are not fully delocalized between the two rings in carbon-based spiroconjugated systems. ${ }^{29,} 30$ Spiropentadiene is the simplest case, constituting two ethene units fused together through a fifth spiro-carbon. Similar to allene, the parent spiropentadiene has $D_{2 d}$ symmetry with four $\pi$-electrons. ${ }^{31}$ A fairly unstable motif due to bond strain, $^{32}$ spiropentadiene has been synthesized with some variation including its sila- and germaanalogues. ${ }^{33-36}$ Shown in the left column of Figure 3a, its HOMO and HOMO- 1 are degenerate and each have clear $\pi$-character on one side of the molecule. The LUMO and LUMO+1 are nondegenerate and the two $\pi$-segments mix due to through-space $\pi$-overlap, which is characteristic of spiroconjugated systems. ${ }^{8}$, 9,28 The disubstitution we performed on allene has a similar effect on spiropentadiene (Figure 3a). The symmetry is reduced from $D_{2 d}$ to $C_{2}$ and the degenerate $\pi_{x}$ and $\pi_{y}$ mix through-bond via the central carbon atom into helical $\pi_{P}$ and $\pi_{M}$. This MOmixing is similar to that seen in saturated triangulanes where helical $\sigma$-orbitals appear, ${ }^{37}$ but in dimethylspiropentadiene $\pi$ orbitals of both helicities are present. The unoccupied $\pi_{x+y}{ }^{*}$ and $\pi_{x-y} *$ are largely unchanged by the methyl substituents. 
The four $\pi \rightarrow \pi^{*}$ transitions in $D_{2 d^{-}}$-spiropentadiene will be two degenerate pairs belonging to the $E$ representation (direct products of the $E$ with $A_{2}$ and $B_{1}$ representations in Figure $\left.3 b\right) .{ }^{24}$, 38, 39 All four $\pi \rightarrow \pi^{*}$ transitions are electric-dipole allowed (belonging to one of the irreducible representation of $\mu_{x, y, z}$ ), and this does not change when the symmetry is reduced by substituents. In dimethylspiropentadiene the electronic transitions become non-degenerate, but do not split by much energetically. Different from dimethylallene, the transitions are not equally weighted linear combinations. Take the two lowest energy MO excitations; $\pi_{P} \rightarrow \pi_{x+y}{ }^{*}$ belong to the $\mathrm{B}$ irreducible representation, while $\pi_{M} \rightarrow \pi_{x+y} *$ belong to $A$. These two excitations are symmetry-protected and cannot mix to form a superposition. Listed in Figure 3c, there is a contribution from higher energy excitations of same symmetry. These excitations into $\pi_{x-y} *$ contribute less than $10 \%$ to the $S_{0} \rightarrow S_{1}$ and $S_{0} \rightarrow S_{2}$ transitions (see Figure $S 2$ for method dependence).

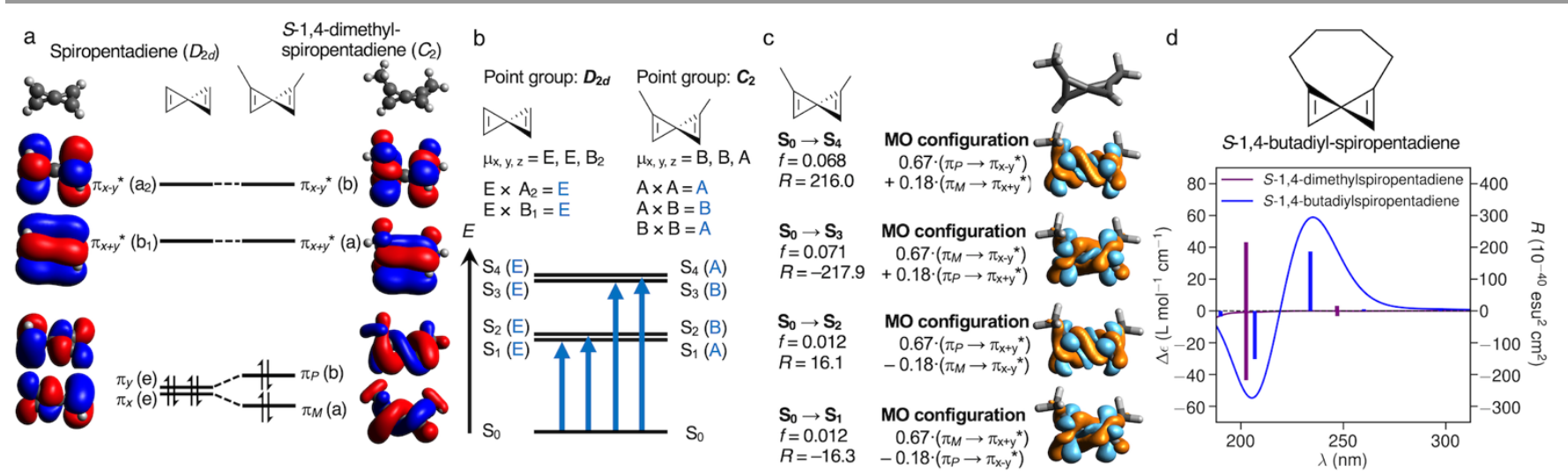

Figure 3. a) HOMO-1 to LUMO+1 of spiropentadiene and S-1,4-dimethylspiropentadiene. b) Overview of their first four electronic transitions, and their symmetry designations. Electric-dipole $\left(\mu_{x, y, z}\right)$ allowed transitions are in blue. c) Change of electron density for the $S_{0} \rightarrow S_{1}, S_{0} \rightarrow S_{2}, S_{0} \rightarrow S_{3}$ and $S_{0} \rightarrow S_{4}$ transitions of $S-1,4-$ dimethylspiropentadiene. d) Simulated ECD spectrum of S-1,4-dimethylspiropentadiene and butadiyl-linked spiropentadiene. Oscillator strengths, $f$, are dimensionless. Rotatory strengths, $R$, are in $10^{40} \mathrm{erg} \cdot \mathrm{esu} \cdot \mathrm{cm} /$ Gauss. Depletion of density is in orange. Computed at the $\omega$ B97X-D/Def2-TZVP level of theory.

As a consequence of their symmetry-protected helicity, properties relating to the electronic transitions will not be limited by the dual-helicity of MOs. The four $\pi-\pi^{*}$ transitions have clear helicity associated to the change of electron density as shown in Figure 3c. The mixing of the two perpendicular $\pi$ systems is thus reflected in the electron density. This is the effect of the through-bond interaction mediated by the spirocarbon, and it is a conclusive outcome of helical $\pi$-conjugation in spiroconjugated molecules.

All four electronic transitions have notable rotatory strengths, in particular the $\mathrm{S}_{0} \rightarrow \mathrm{S}_{3}$ and $\mathrm{S}_{0} \rightarrow \mathrm{S}_{4}$ transitions. Each pair of neardegenerate transitions have opposite helicity in the change of electron density and opposite sign of the rotatory strength. The rotatory strength of each transition describes the difference in the absorption rate of left- and right-polarized light. Consequently, circularly polarized light will be more likely to absorb due to transitions of a specific helicity. This result may open the possibility for imaging the helical electron density arising directly from a unique MO using photoemission tomographic imaging if circularly polarized light is applied. ${ }^{40,41}$ The total chiroptical response of dimethylspiropentadiene will be small due to the opposite signs in the rotatory strengths, because the optical activity is experimentally observed as the difference in absorption of left- and right-polarized light $(\Delta \varepsilon)$. This is clear from the simulated electronic circular dichroism (ECD) spectrum shown in purple in Figure 3d. The two sets of near-degenerate transitions in dimethylspiropentadiene have large rotatory strengths but $\Delta \varepsilon$ remain almost zero.

To split the transitions energetically, we examine a strained spiropentadiene by applying a cyclically connected butadiyl- substituent as illustrated in Figure $3 d$. This system assumes a chair-like conformation, which alters both the dihedral and bond angles of the spiro-segment. This strain alters the energetics of the frontier MOs considerably by unwinding or overwinding the helices. ${ }^{13}, 21$ The chiroptical response is increased in S-1,4-butadiylspiropentadiene and the equivalently constrained allene (see Figure S6 and S7). This strained spiropentadiene species sees has a strong chiroptical response compared to the dimethylspiropentadiene. The big rotatory strengths of the $S_{0} \rightarrow S_{3}$ and $S_{0} \rightarrow S_{4}$ transitions now give rise to two opposite peaks in the simulated ECD spectrum shown in Figure $3 \mathrm{~d}$. The extra strain is likely to destabilize the molecule. However, it serves as a proof-of-concept for how the structure of spiroconjugated molecules can be manipulated to maximize the chiroptical response.

The analysis we have made here for spiropentadiene is also valid for its silicon and germanium analogues. Helical $\pi$-MOs appear in spiropentasiladienes and spiropentagermadienes, and the lowest-lying $\pi \rightarrow \pi^{*}$ transitions have helical change of density. Different from the carbon systems, there are also $\sigma$ MOs close to the HOMO-LUMO gap which contribute to the electronic transitions and limit the helicity. A full overview is provided in Figure S8. These results all demonstrate how perpendicular $\pi$-system mix into helical ones in spiroconjugated molecules and their analogues. In conjunction with recent experimental work by Honda et al. on electron delocalization between the perpendicular $\pi$-systems in spiropentasiladiene, ${ }^{7}$ it seems clear that helical $\pi$-conjugation has experimentally appreciable consequences in small spiroconjugated systems. 
We have made a brief assessment of the extent to which helical $\pi$-conjugation can also be present in larger spiroconjugated motives and tricyclic analogues. ${ }^{39}$ In spirononatetraene there are eight $\pi$-electrons and the MO symmetries differ from spiropentadiene. Though the HOMO-2 and HOMO-3 of dimethylspirononatetraene are clearly helical (Figure S9), they are less relevant for observable properties. Tricyclic spiroanalogues where the parent system has $D_{2 d}$ symmetry will also have similar mixing of its two $\pi$-systems. However, there will be additional nodal planes due to the extra $\sigma$-bonds (Figure S11 and S12). ${ }^{10,11,42}$ The electrohelicity effect thus appears to be less pronounced in larger systems.

In summary, we have explored how helical $\pi$-conjugation between the perpendicular $\pi$-systems of allene and spiropentadiene affect their electronic transitions and the helicity thereof. In allene the helicity of the $\pi$-MOs cancel out as all transitions are linear combinations of excitations involving MOs of both helicities. In spiropentadiene, the HOMO-1 and HOMO become helical in similar fashion to the $\pi$-mixing in allene, but the LUMO and LUMO+1 remain rectilinear $\pi$-MOs. The $\pi \rightarrow \pi^{*}$ transitions are dominated by a single helical configuration, which is symmetry-protected from mixing with excitations of opposite helicity. These transitions have clear helicity associated to the change of electron density. Different from allene, the electrohelicity effect is connected to the optical properties of spiropentadiene. The sign of the rotatory strength of each transition correlates with the helicity. The properties of substituted spiropentadienes are not limited by the dualhelicity of the $\pi$-MOs, and thus hold potential for spectroscopic verification of helical $\pi$-conjugation.

The authors thank Maria Fumanal (EPFL) for technical help with CASPT2 computations. MHG is grateful for funding from Independent Research Fund Denmark, case no. 9056-00009B.

\section{Conflicts of interest}

There are no conflicts to declare.

\section{References}

1. E. Soriano and I. Fernandez, Chem. Soc. Rev., 2014, 43, 30413105.

2. D. Wendinger and R. R. Tykwinski, Acc. Chem. Res., 2017, 50, 1468-1479.

3. M. H. Garner, R. Hoffmann, S. Rettrup and G. C. Solomon, ACS Cent. Sci., 2018, 4, 688-700.

4. H. Fischer and H. Kollmar, Theor. Chim. Acta, 1968, 12, 344348.

5. H. E. Zimmerman, Acc. Chem. Res., 1971, 4, 272-280.

6. R. Herges, J. Org. Chem., 2015, 80, 11869-11876.

7. S. Honda, R. Sugawara, S. Ishida and T. Iwamoto, J. Am. Chem. Soc., 2021, 143, 2649-2653.

8. H. E. Simmons and T. Fukunaga, J. Am. Chem. Soc., 1967, 89, 5208-5215.

9. R. Hoffmann, A. Imamura and G. D. Zeiss, J. Am. Chem. Soc., 1967, 89, 5215-5220.

10. P. Bischof, R. Gleiter and R. Haider, J. Am. Chem. Soc., 1978, 100, 1036-1042.

11. R. Gleiter, R. Haider, J. Spanget-Larsen and P. Bischof, Tetrahedron Lett., 1983, 24, 1149-1152.
12. C. H. Hendon, D. Tiana, A. T. Murray, D. R. Carbery and A. Walsh, Chem. Sci., 2013, 4, 4278-4284.

13. A. Imamura and Y. Aoki, Chem. Phys. Lett., 2013, 590, 136140.

14. T. Xu, J. H. Li, R. Momen, W. J. Huang, S. R. Kirk, Y. Shigeta and S. Jenkins, J. Am. Chem. Soc., 2019, 141, 5497-5503.

15. M. H. Garner and C. Corminboeuf, Org. Lett., 2020, 22, 80288033.

16. Y. Orimoto, Y. Aoki and A. Imamura, J. Phys. Chem. C, 2019, 123, 11134-11139.

17. M. H. Garner, A. Jensen, L. O. H. Hyllested and G. C. Solomon, Chem. Sci., 2019, 10, 4598-4608.

18. M. Caricato, J. Chem. Theory Comput., 2015, 11, 1349-1353.

19. M. D. Peeks, P. Neuhaus and H. L. Anderson, Phys. Chem. Chem. Phys., 2016, 18, 5264-5274.

20. A. Ozcelik, D. Aranda, S. Gil-Guerrero, X. A. Pola-Otero, M. Talavera, L. Wang, S. K. Behera, J. Gierschner, Á. Peña-Gallego, F. Santoro, R. Pereira-Cameselle and J. L. Alonso Gómez, Chem. Eur. J., 2020, 26, 17342-17349.

21. M. H. Garner, W. Bro-Jørgensen and G. C. Solomon, J. Phys. Chem. C, 2020, 124, 18968-18982.

22. S. Gunasekaran and L. Venkataraman, J. Chem. Phys., 2020, 153, 124304.

23. P. Pinter and D. Munz, J. Phys. Chem. A, 2020, 124, 1010010110.

24. F. A. Cotton, Chemical Applications of Group Theory, John Wiley \& Sons, Inc., New York, 3rd edn., 1990.

25. A. Rauk, A. F. Drake and S. F. Mason, J. Am. Chem. Soc., 1979, 101, 2284-2289.

26. C. J. Elsevier, P. Vermeer, A. Gedanken and W. Runge, J. Am. Chem. Soc., 1985, 107, 2537-2547.

27. K. B. Wiberg, Y.-g. Wang, S. M. Wilson, P. H. Vaccaro, W. L. Jorgensen, T. D. Crawford, M. L. Abrams, J. R. Cheeseman and M. Luderer, J. Phys. Chem. A, 2008, 112, 2415-2422.

28. R. Gleiter and W. Schaefer, Acc. Chem. Res., 1990, 23, 369375.

29. H. S. Rzepa and K. R. Taylor, J. Chem. Soc., Perkin Trans. 2, 2002, 1499-1501.

30. D. Hall and H. S. Rzepa, Org. Biomol. Chem., 2003, 1, 182185.

31. M. C. Böhm and R. Gleiter, J. Chem. Soc., Perkin Trans. 2, $1979,443-448$.

32. J. P. Kenny, K. M. Krueger, J. C. Rienstra-Kiracofe and H. F. Schaefer, J. Phys. Chem. A, 2001, 105, 7745-7750.

33. W. E. Billups and M. M. Haley, J. Am. Chem. Soc., 1991, 113, 5084-5085.

34. R. K. Saini, V. A. Litosh, A. D. Daniels and W. E. Billups, Tetrahedron Lett., 1999, 40, 6157-6158.

35. T. Iwamoto, M. Tamura, C. Kabuto and M. Kira, Science, 2000, 290, 504.

36. Y. Guo, Z. Xia, J. Liu, J. Yu, S. Yao, W. Shi, K. Hu, S. Chen, Y. Wang, A. Li, M. Driess and W. Wang, J. Am. Chem. Soc., 2019, $141,19252-19256$.

37. A. de Meijere, A. F. Khlebnikov, R. R. Kostikov, S. I.

Kozhushkov, P. R. Schreiner, A. Wittkopp and D. S. Yufit, Angew. Chem. Int. Ed., 1999, 38, 3474-3477.

38. C. Batich, E. Heilbronner, E. Rommel, M. F. Semmelhack and J. S. Foos, J. Am. Chem. Soc., 1974, 96, 7662-7668.

39. H. Dürr and R. Gleiter, Angew. Chem. Int. Ed. Engl., 1978, 17, 559-569.

40. J. Itatani, J. Levesque, D. Zeidler, H. Niikura, H. Pépin, J. C. Kieffer, P. B. Corkum and D. M. Villeneuve, Nature, 2004, 432, 867.

41. M. Wießner, D. Hauschild, C. Sauer, V. Feyer, A. Schöll and F. Reinert, Nat. Commun., 2014, 5, 4156.

42. J. Dressel, K. L. Chasey and L. A. Paquette, J. Am. Chem. Soc., $1988,110,5479-5489$ 